\title{
Search for double beta decay with the NEMO-3 experiment
}

\author{
Vera Beillet-Kovalenko* on behalf of the NEMO collaboration \\ IPHC, Université de Strasbourg, CNRS/IN2P3, F-67037, Strasbourg, France \\ and Joint Institute for Nuclear Research, 141980 Dubna, Russia \\ E-mail: vera.kovalenko@ires.in2p3.fr
}

The NEMO-3 experiment is searching for neutrinoless double beta decay. The experiment has been taking data since 2003 with seven isotopes. Data up to the end of 2008 show no evidence for neutrinoless double beta decay which permits setting at the $90 \% \mathrm{CL}$, lower limits on the transitions: $T_{1 / 2}^{0 v}>1.1 \cdot 10^{24}$ y for ${ }^{100} \mathrm{Mo}$ and $T_{1 / 2}^{0 v}>3.6 \cdot 10^{23}$ y for ${ }^{82} \mathrm{Se}$. NEMO-3 also measures two-neutrino double beta decays for other isotopes and has reached the highest precision measurements to date.

The 2009 Europhysics Conference on High Energy Physics,

July 16 - 222009

Krakow, Poland

${ }^{*}$ Speaker. 
Introduction. Neutrinoless double beta decay $(0 v \beta \beta)(A, Z) \rightarrow(A, Z+2)+2 \mathrm{e}^{-}$, unlike the twoneutrino double beta decay $(\mathrm{A}, \mathrm{Z}) \rightarrow(\mathrm{A}, \mathrm{Z}+2)+2 \mathrm{e}^{-}+2 \bar{v}$, supposes the violation of the lepton number by two and a Majorana neutrino type. In the mass mechanism decay model [1] an exchange of neutrino helicity is needed and occurs only if the neutrinos are massive Majorana particles. The decay half-life of such a mechanism is a function of the phase space factor, the nuclear matrix element (NME) and the effective neutrino mass $\left\langle m_{v}\right\rangle$. Therefore the observation of $0 v \beta \beta$ decay would indicate the nature of the neutrino and give access to its mass.

NEMO-3 detector. The NEMO-3 experiment ${ }^{1}$ is currently running and located in the underground laboratory $\mathrm{LSM}^{2}$ in the Fréjus tunnel between France and Italy. The design of the NEMO-3 detector is for the direct detection of two electrons from double beta decay by a tracking chamber and a calorimeter (Fig. 1). A full description of the detector can be found in [2]. Together with
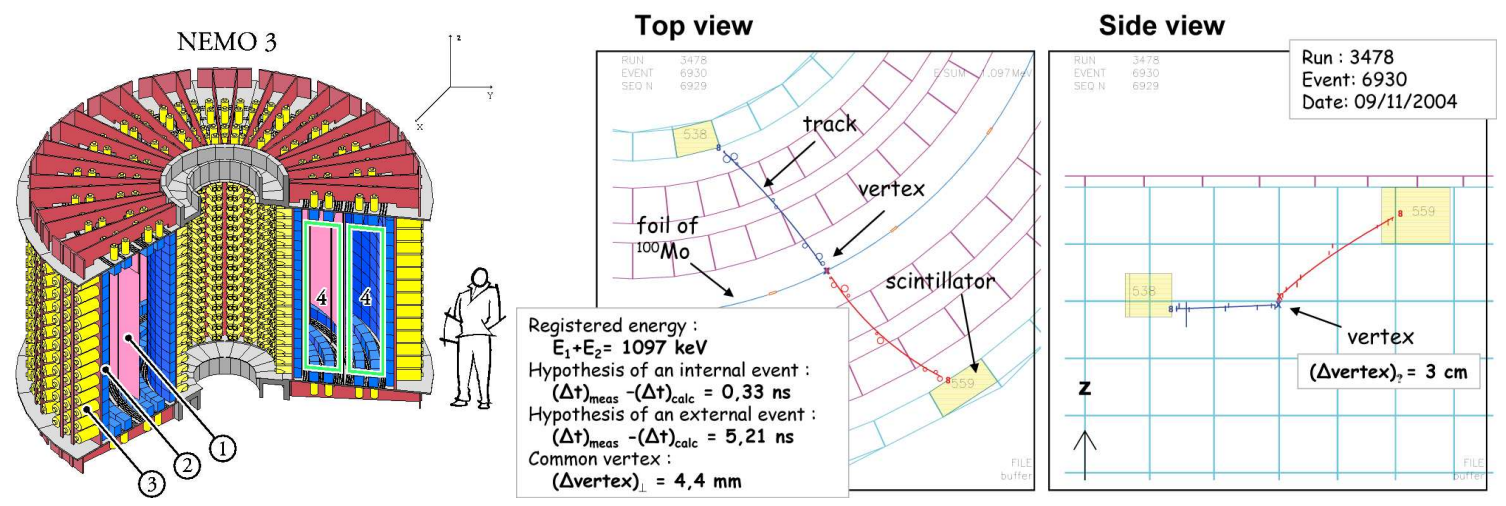

Figure 1: On the left side: general view of the NEMO-3 detector with 1) source foils, 2) plastic scintillator blocks, 3) photomultiplier tubes, 4) tracking volume. On the right side: example of an event candidate from two-neutrino double beta decay of ${ }^{100}$ Mo.

source foils of ${ }^{100} \mathrm{Mo}(7 \mathrm{~kg})$ and ${ }^{82} \mathrm{Se}(1 \mathrm{~kg})$ which are used to search for $0 v \beta \beta$ decay, smaller amounts of ${ }^{116} \mathrm{Cd},{ }^{150} \mathrm{Nd},{ }^{96} \mathrm{Zr},{ }^{48} \mathrm{Ca}$ and ${ }^{130} \mathrm{Te}$ are used to measure two-neutrino double beta decay (see Table 1). The sectors with pure copper, natural and enriched tellurium are used to study the external background.

A typical double beta decay candidate is shown in Fig. 1. The tracking program finds two electron tracks (shown in red and blue) with a common vertex in a source foil. Each track ends as it enters a plastic scintillator and must deposit an energy greater than $200 \mathrm{keV}$. In order to reject external background events a time-of-flight analysis is used.

A critical issue of the NEMO-3 experiment is the background. The energy region around $3 \mathrm{MeV}$, which is the decay energy of ${ }^{100} \mathrm{Mo}$ and ${ }^{82} \mathrm{Se}$, is shared with some energetic natural radioactivity that can create two electrons in the source foil and mimic $0 v \beta \beta$ decay. A distinctive advantage of the NEMO-3 experiment is its capability to measure the backgrounds [3]. In order to reduce the radon background contribution observed at the beginning of the NEMO-3 experiment, a radon trapping facility was installed in the laboratory and the detector was covered with a Mylar

\footnotetext{
${ }^{1}$ NEMO: Neutrino Ettore Majorana Observatory

${ }^{2}$ Laboratoire Souterrain de Modane
} 
tent. They reduce the radon level in the tracking chamber by a factor of approximately six. Therefore the analyzed data have been divided into two periods, Phase 1 and Phase 2, before and after the radon facility was installed.

\begin{tabular}{c|c|c|c|ll}
\hline Isotope & Mass $(\mathrm{g})$ & $\mathrm{Q}_{\beta \beta}(\mathrm{keV})$ & Signal/Bkg & $\mathrm{T}_{1 / 2}\left[10^{19}\right.$ years $]$ & \\
\hline${ }^{100} \mathrm{Mo}$ & 6914 & 3034 & 40 & $0.711 \pm 0.002($ stat $) \pm 0.054$ (syst) & {$[4]$} \\
${ }^{100} \mathrm{Mo}\left(0_{1}^{+}\right)$ & & & 3 & $57_{-9}^{+13}($ stat $) \pm 8($ syst $)$ & {$[5]$} \\
${ }^{82} \mathrm{Se}$ & 932 & 2995 & 4 & $9.6 \pm 0.3($ stat $) \pm 1.0($ syst $)$ & {$[4]$} \\
${ }^{116} \mathrm{Cd}$ & 405 & 2805 & 7.5 & $2.8 \pm 0.1$ (stat) $\pm 0.3($ syst $)$ & \\
${ }^{150} \mathrm{Nd}$ & 37.0 & 3367 & 2.8 & $0.911_{-0.022}^{+0.025}$ (stat) \pm 0.063 (syst) & {$[6]$} \\
${ }^{96} \mathrm{Zr}$ & 9.4 & 3350 & 1 & $2.35 \pm 0.14($ stat $) \pm 0.16$ (syst) & {$[7]$} \\
${ }^{48} \mathrm{Ca}$ & 7.0 & 4272 & 6.8 & $4.4_{-0.4}^{+0.5}($ stat $) \pm 0.4($ syst $)$ & \\
${ }^{130} \mathrm{Te}$ & 454 & 2529 & 0.35 & $69 \pm 9($ stat $) \pm 10$ (syst) & \\
\hline
\end{tabular}

Table 1: NEMO-3 results for the half-life of two-neutrino double beta decay measurements.

Two-neutrino double beta decay measurements. The NEMO-3 detector is unique in the sense that it is able to measure the total electron energy, the energy of each electron and angular distribution between the electrons. The two-neutrino double beta decay $(2 v \beta \beta)$ is a second order process of the weak interactions. A good knowledge of this process is critical to the $0 v \beta \beta$ decay spectra as it contributes an unavoidable background. The precise measurements of the half-life and event kinematics are used to constrain the model to calculate the NME for $0 v \beta \beta$ decay. Table 1 summarizes the results of the half-life measurements for the isotopes present in NEMO-3.

Neutrinoless double beta decay search. The $0 v \beta \beta$ decay is mediated by light Majorana neutrino exchange which leads to a peak at the endpoint energy of the two electrons, smeared by the energy resolution of the detector, as shown in Fig. 2. The analysis of the data taken up to the end of
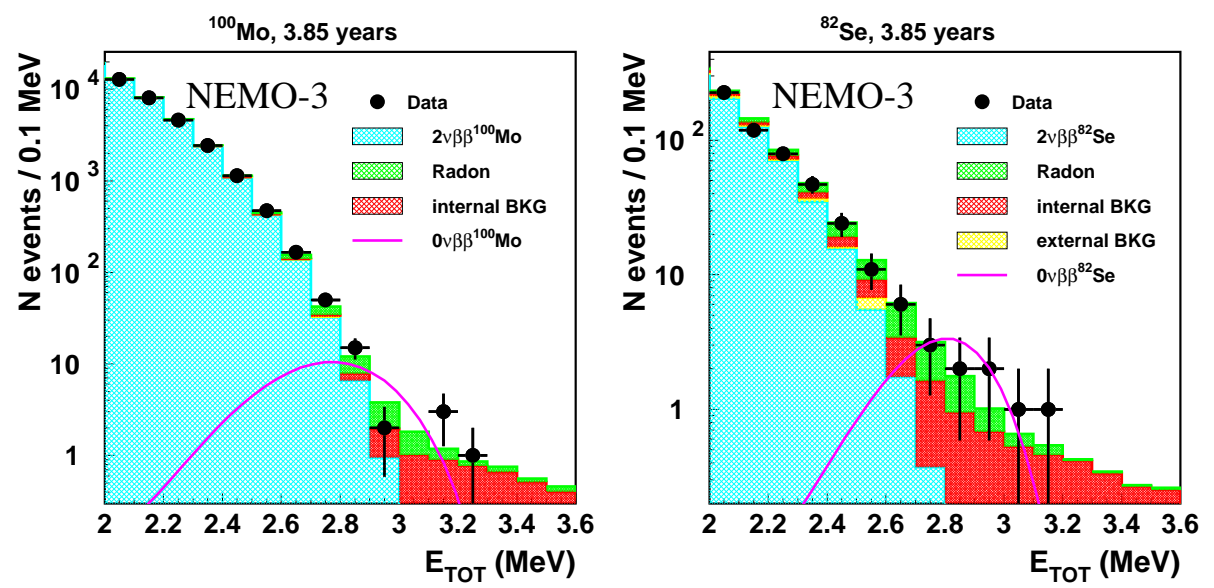

Figure 2: High energy tail of the energy sum distribution for events in molybdenum (left) and selenium (right) foils are shown with black points. The background contributions are shown within the histogram. The shape of a hypothetical signal is shown by the magenta curve in arbitrary units.

2008 is shown with no signal observed in the molybdenum or selenium. Therefore lower limits on 
the decay half-lifes are $\mathrm{T}_{1 / 2}^{0 v}>1.1 \cdot 10^{24} \mathrm{y}(90 \% \mathrm{CL})$ and $\mathrm{T}_{1 / 2}^{0 v}>3.6 \cdot 10^{23} \mathrm{y}(90 \% \mathrm{CL})$, respectively. These numbers translate into a range of upper limits on the effective Majorana mass of $\left\langle m_{v}\right\rangle<$ $0.45-0.93 \mathrm{eV}$ for ${ }^{100} \mathrm{Mo}$ and $\left\langle m_{v}\right\rangle<0.89-1.61 \mathrm{eV}$ for ${ }^{82} \mathrm{Se}$, according to the most recent NME calculations used by NEMO-3 $[9,10,11]$.

Search for right handed currents and Majorana emission. Other exotic mechanisms such as right-hand currents or Majoron emission could also contribute to $0 v \beta \beta$ decay. The latter would yield a different shape for the two electron energy sum distribution than the one from $2 v \beta \beta$ decay [12]. A maximum likelihood analysis of the deviation of the energy shape from the calculated $2 v \beta \beta$ decay shape was performed. The resulting limits on the half-lives, couplings for righthanded currents and different Majoron spectral indices "n" are shown in Table 2.

\begin{tabular}{|c|l|l|c|c|c|}
\hline Isotope & $\mathrm{T}_{1 / 2}^{0 v \beta \beta}(\mathrm{y}), \mathrm{V}+\mathrm{A}$ & \multicolumn{3}{|c|}{$\mathrm{T}_{1 / 2}^{0 v \beta \beta}(\mathrm{y})$, Majoron(s) emission } \\
\cline { 3 - 6 } & & $\mathrm{n}=1$ & $\mathrm{n}=2$ & $\mathrm{n}=3$ & $\mathrm{n}=7$ \\
\hline${ }^{100} \mathrm{Mo}$ & $>5.7 \cdot 10^{23}$ & $>2.7 \cdot 10^{22}$ & $>1.7 \cdot 10^{22}$ & $>1.0 \cdot 10^{22}$ & $>7 \cdot 10^{19}$ \\
& $\lambda<1.4 \cdot 10^{-6}$ & $\mathrm{~g}_{e e}<(0.4-1.8) \cdot 10^{-4}$ & & & \\
\hline${ }^{82} \mathrm{Se}$ & $>2.4 \cdot 10^{23}$ & $>1.5 \cdot 10^{22}$ & $>6.0 \cdot 10^{21}$ & $>3.1 \cdot 10^{22}$ & $>5 \cdot 10^{20}$ \\
& $\lambda<2.0 \cdot 10^{-6}$ & $\mathrm{~g}_{e e}<(0.7-1.9) \cdot 10^{-4}$ & & & \\
\hline
\end{tabular}

Table 2: Limits on half-life and coupling constant measurements for $\mathrm{V}+\mathrm{A}$ and Majoron emission. The NME from [13] were used to calculate the coupling constants $\lambda$.

\section{References}

[1] T. Tomoda, Rep. Prog. Phys. 54, 53-126 (1991).

[2] R. Arnold et al, Nucl. Instrum. Methods A 536, 79 (2005).

[3] J. Argyriades et al, Nucl. Instrum. Methods A 606, 449 (2009).

[4] R. Arnold et al, Phys. Rev. Lett. 95, 182302 (2005).

[5] R. Arnold et al, Nucl. Phys. A 781, 209 (2007).

[6] J. Argyriades et al, arXiv:0810.0248 [hep-ex] (2008), accepted by Phys. Rev. C. i

[7] J. Argyriades et al, arXiv:0906.2694 [nucl-ex] (2009).

[8] V.Vasiliev, arXiv:0710.5090v1 [nucl-ex] (2007).

[9] M. Kortelainen and J. Suhonen, Phys. Rev. C 76, 024315 (2007).

[10] M. Kortelainen and J. Suhonen, Phys. Rev. C 75, 051303(R) (2007).

[11] F. Šimkovich et al, Phys. Rev. C 77, 045503 (2008).

[12] R. Arnold et al, Nucl. Phys. A 765, 483 (2006).

[13] M. Aunola and J. Suhonen,Nucl. Phys. A 643, 207 (1998), J. Suhonen, Nucl. Phys. A 700, 649 (2002) 